\title{
Mechanisms and Consequences of Anthropomorphizing Autonomous Products
}

\section{The Role of Schema Congruity and Prior Experience}

\author{
Moritz Jörling1 ${ }^{\mathbb{D}} \cdot$ Robert Böhm $^{2} \cdot$ Stefanie Paluch $^{1,3}$
}

Received: 29 October 2019 / Accepted: 17 August 2020 / Published online: 15 September 2020

(C) The Author(s) 2020

\begin{abstract}
In the present research, we test the mechanisms (Studies 1a and 1b, conducted online), consequences, and limitations (Study 2, conducted in the lab) of anthropomorphizing autonomous (vs. manual) products. Building on previous theoretical and empirical research on product anthropomorphism, we argue and find that anthropomorphism is perceived to be more congruent with autonomous products than with manual products. Furthermore, we show that anthropomorphism increases the liking of autonomous products, given that consumers have no prior experience with autonomous products. Increased liking of autonomous products due to anthropomorphism, in turn, increases purchase intentions and positive evaluations of outcomes obtained by the autonomous product. The findings are discussed with regard to optimal marketing and design of autonomous products.
\end{abstract}

Keywords Product autonomy $\cdot$ Anthropomorphism $\cdot$ Schema congruity $\cdot$ User experience $\cdot$ Product Liking

Electronic supplementary material The online version of this article (https://doi.org/10.1007/ s41464-020-00100-3) contains supplementary material, which is available to authorized users.

M. Jörling

joerling@time.rwth-aachen.de

1 Service and Technology Marketing, School of Business and Economics, RWTH Aachen University, Kackertstraße 7, 52072 Aachen, Germany

2 Department of Psychology, Department of Economics, and Copenhagen Center for Social Data Science (SODAS), University of Copenhagen, Øster Farimagsgade 2A, 1353 Copenhagen, Denmark

3 Centre for Relationship Marketing and Service Management, Department of Marketing, Hanken School of Economics, Helsinki, Finland 
Autonomous products are on the rise. With no human interference, these products undertake an increasing number of tasks that had to be executed by humans in the past. In domestic and professional environments, they clean floors and windows, cut the grass, or transport goods from one place to another. It is projected that 32.4 million units of autonomous products only for domestic tasks will be sold by 2020 , representing a market value of $\$ 11.3$ billion and a growth rate of 30-35\% per year (Executive Summary World Robotics 2019 Service Robots, 2019). Additionally, a study by the Boston Consulting Group in collaboration with the World Economic Forum projects for 2035 that $25 \%$ of the car market worldwide will consist of partially and fully autonomous vehicles ("Autonomous Vehicle Adoption Study" BCG 2017).

Due to the increasing dissemination of autonomous products, developing successful marketing and product strategies that take into account consumers' needs and expectations are becoming increasingly relevant. This addresses both the advertisement and design of autonomous products in order to increase consumers' pre-purchase liking as well as their post-purchase satisfaction with the product. In fact, understanding individuals' perceptions of autonomous products and its consequences for product evaluation and liking has been identified as one of the potentially most growing topics of future consumer research (Rijsdijk and Hultink 2003, 2009; Schweitzer and van den Hende 2016).

A prominent strategy of product marketing is the anthropomorphization of products. Previous research has shown that consumers perceive anthropomorphized products more positively than non-anthropomorphized products (e.g., Chandler and Schwarz 2010; Kim and McGill 2011; Touré-Tillery and McGill 2015). However, little is known whether anthropomorphizing autonomous products also increases product liking (Belk 2016; Goudey and Bonnin 2016) and, additionally, whether this has secondary effects on purchase intentions and evaluations of the product's performance. In the present paper, we therefore theoretically analyze why and under what conditions anthropomorphization could affect the liking of autonomous products. Building on schema congruity theory (Fiske and Linville 1980), we argue that autonomous products are a particularly suitable product category to apply anthropomorphization in order to increase product liking. We also identify potential boundary conditions of this effect, i.e., prior experience with autonomous products. We further hypothesize that increased product liking due to anthropomorphizing autonomous products may lead to greater purchase intentions and more positive evaluations of the autonomous product's task performance. We test and support our hypotheses in two online studies (Studies 1a and 1b) and one lab study (Study 2).

Thus, our research contributes to the literature of schema congruity (Aggarwal and McGill 2007; Fiske and Linville 1980; Mandler 1982; Meyers-levy and Tybout 1989) and anthropomorphism (Aggarwal and McGill 2007; Epley et al. 2007; Waytz et al. 2014) by applying it to the growing field of autonomous products. In this vein, we demonstrate that there are important conceptual differences in the effects of anthropomorphizing autonomous vs. manual products. A more nuanced theoretical and empirical overview leading to our hypotheses is provided in the next sections. 


\section{Product Autonomy}

Autonomy, which is the capability to carry out its own processes and operations (Beer et al. 2014), can consist in virtual systems (often called agents) as well as in physical products (often called robots) (Bekey 1998). The degree of a physical product's autonomy, which exists on a continuum from no autonomy to full autonomy, is determined by sensors and actuators, embedded in the particular product (Ziemke 2008). The degree of autonomy can be defined as the extent to which the product "can sense its environment, plan based on that environment, and act upon that environment with the intent of reaching some task-specific goal (either given to or created by the robot) without external control." (Beer et al. 2014, p. 77). For investigations in a consumer context, the level of the product's autonomy is of utmost importance, since it determines the tasks the product is able to perform and therefore the level and frequency of interaction the product has with human operators (Beer et al. 2014). The level and the frequency of consumers' interaction with the product, in turn, influence consumers' perception of the product. For example, consumers usually prefer a medium level of product autonomy, which reduces discomfort by overtaking monotonous work tasks, but which still leaves the option for interaction and decisions taken by the consumer her-/himself (Rijsdijk and Hultink 2003). It has been shown that a lack of interaction between consumer and an autonomous product increases perceived disempowerment of the consumer, which in turn decreases the intention to adopt the product (Schweitzer and van den Hende 2016). Moreover, when consumers interact with autonomous products, they usually apply a social model to explain and understand the behavior of the product (Breazeal 2003). As a consequence, in combination with other characteristics of autonomous products (e.g., human-like pace in movement), consumers might also perceive anthropomorphic qualities in autonomous products (Aggarwal and McGill 2007; Epley et al. 2007; Goudey and Bonnin 2016). Hence, in the following section we review the literature on the relation between (autonomous) technologies and anthropomorphism.

\section{Anthropomorphism and Technology}

Anthropomorphism refers to the attribution of human traits, behavioral characteristics, or even emotional states to nonhuman agents or objects (Epley et al. 2007) and "has a major impact on human behavior [and] choices" (Złotowski et al. 2015, p. 347). Humans have a natural tendency to anthropomorphize non-human objects, for instance, by seeing human shapes in nature or creating miraculous creatures with human traits (Waytz et al. 2010a). Psychologists and anthropologists explain this proneness to anthropomorphize objects as means of increasing the feeling of predicting the object's future behavior, thereby reducing uncertainty (Waytz et al. 2010b).

Consumer behavior research has primarily focused on effects of anthropomorphized brands (Aaker 1997; Aggarwal and McGill 2012; Fournier and Alvarez 2012). Yet, marketers and product designers may also encourage anthropomorphism 


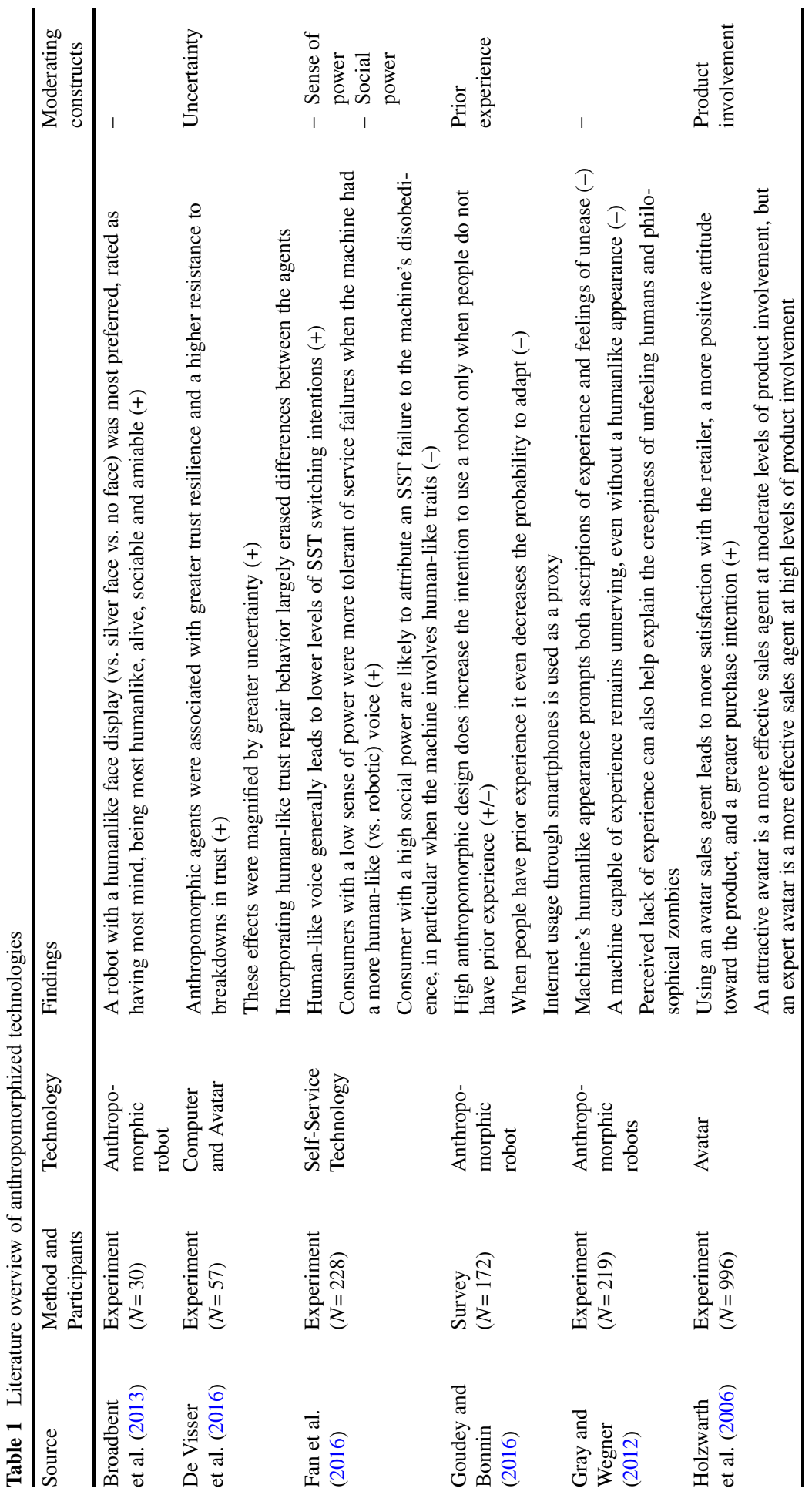




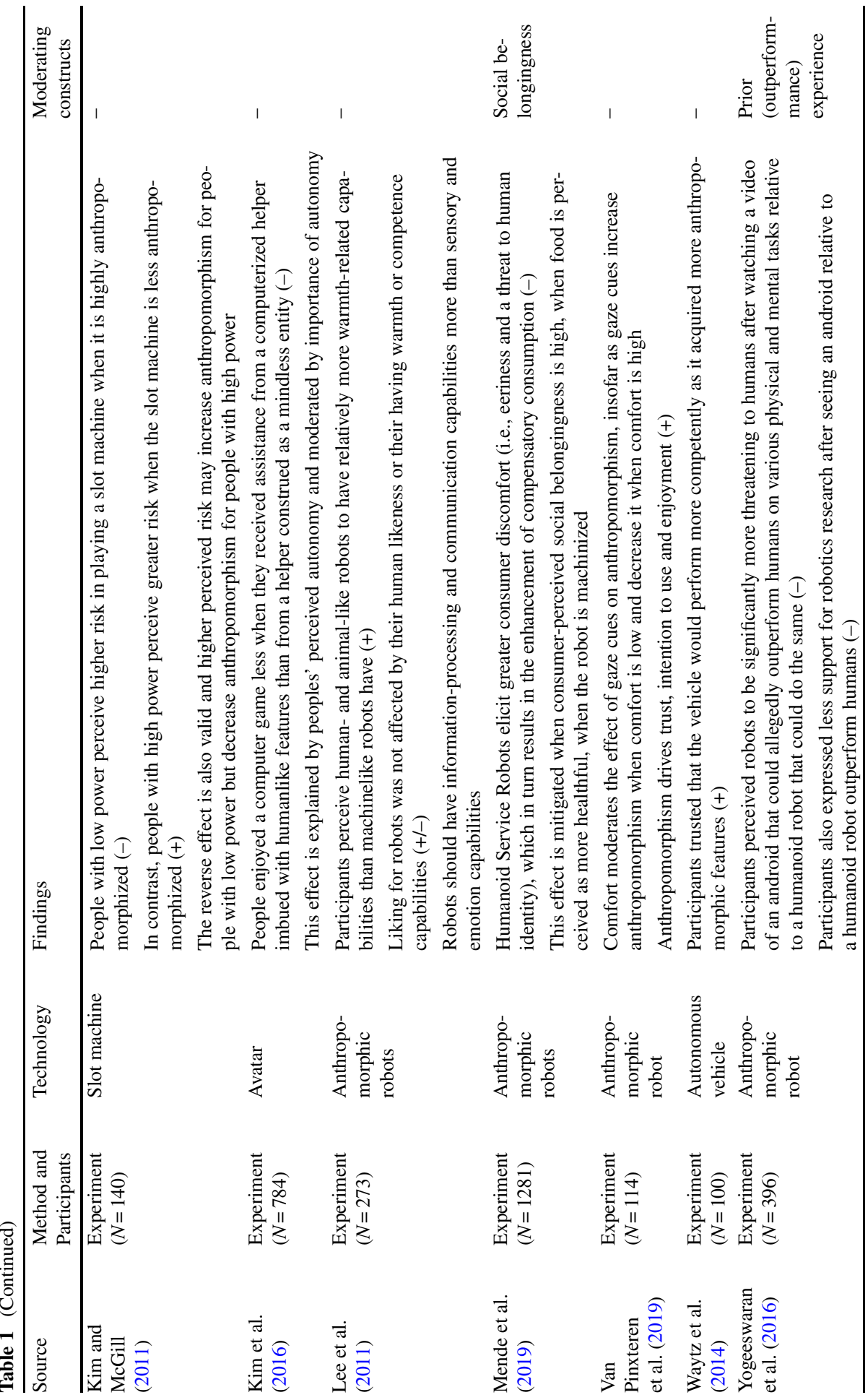


of products. For instance, friendly or aggressive facial expressions in product shapes of, for instance, cars, cell phones, or computer interfaces increase liking of the product (Burgoon et al. 2000; Gong 2008; Koda and Maes 1996) and may even have an impact on sales (Landwehr et al. 2011) or the willingness to replace a product (Chandler and Schwarz 2010). Anthropomorphism of products may also affect how individuals perceive the risk and control of products (Kim and McGill 2011). Therefore, anthropomorphism is a design approach that has been adapted to technologies to overcome potential technology aversions and perceived lack of control (Jörling et al. 2019). However, when considering research from management, marketing, and psychology journals on this topic (see Table 1), the impact of anthropomorphism on consumers' perception of technology is mixed (Goudey and Bonnin 2016; Kim and McGill 2011; Lee et al. 2011; Mende et al. 2019).

Some investigations find a positive impact of anthropomorphism on technology perceptions, like increased trust (Hancock et al. 2011; Waytz et al. 2014), increased liking (Broadbent et al. 2013), or higher failure tolerance (Fan et al. 2016). Other papers, in contrast, find a rather negative relation, like increased eeriness (Gray and Wegner 2012) or higher perceived risk to use a machine (Kim and McGill 2011). And still other research does not find an effect of anthropomorphism on liking of technology at all (Lee et al. 2011). So, what explains these contradictory findings?

First, results differ depending on the investigated technology (Fan et al. 2016). Previous research has mainly investigated professional robots of which some had a low level of product autonomy (Fan et al. 2016; Kim and McGill 2011) or which already had an anthropomorphic design by construction (Goudey and Bonnin 2016; Mende et al. 2019). Domestic robots, which represent the majority of robots that consumers interact with in everyday life, are usually constructed in a non-anthropomorphized way. These products have been widely ignored in previous research.

Second, there could be hidden moderators that need to be considered (Kim and McGill 2011). We propose that the consumers' prior experience with the technology could be of particular relevance. Prior experience is a superordinate construct, which should affect other moderating constructs, such as uncertainty (de Visser et al. 2016) or perceived power in interactions with technology (Fan et al. 2016). However, the construct has been mainly neglected by prior research or was included only as a proxy (i.e., "internet usage through smartphones"; Goudey and Bonnin 2016). Therefore, we investigate prior experience with the technology as a relevant construct, potentially influencing the effect of the schema that may be triggered in users interacting with autonomous products (Epley et al. 2007).

\section{Schema Congruity}

A schema is a framework which covers knowledge of and information about topics, concepts, or categories (Aggarwal and McGill 2007; Fiske and Linville 1980). In a simple way, a schema can be defined as a concept that "describes how data fit an existing pattern" (Fiske and Linville 1980, p. 552). Congruity is given when attributes of a product or stimulus match the relevant schema (Meyers-levy and 
Tybout 1989), such that a given product information corresponds to consumers' expectations (Peracchio and Tybout 1996).

What are the implications of schema congruity when it comes to liking of anthropomorphized autonomous vs. manual products? As described above, autonomous products are characterized as being largely self-reliant, independent in their movement and/or decision-making, work target-oriented, and are often described as intelligent (Goudey and Bonnin 2016). Consider the example of an autonomous vacuum cleaner. This device moves at a human-like pace around the floor and independently makes decisions about where to go, giving the impression of basic cognitive abilities like these of human beings. In contrast, the manual counterpart, i.e., a standard vacuum cleaner, appears rather dumb and lifeless, becoming useful only with active human support. Accordingly, it could be expected that actively projecting "humanlike properties, characteristics, or mental states" (Epley et al. 2007, p. 865) to autonomous products and, therefore, anthropomorphizing them (Breazeal 2003; Epley et al. 2007; Waytz et al. 2014; Złotowski et al. 2015) will be perceived as more congruent with these products' characteristics compared with manual products (Aggarwal and McGill 2007). We therefore hypothesize:

H1: Autonomous products are perceived as more congruent with anthropomorphism than their manual counterparts.

The relation between schema congruity and product liking has been extensively investigated in the marketing literature (Aggarwal and McGill 2007; Mandler 1982; Meyers-levy and Tybout 1989; Peracchio and Tybout 1996). Theory proposes "schema congruity leads to a favorable response because, other things being equal, people like objects that conform to their expectations and allow predictability" (Meyers-levy and Tybout 1989, p. 40). Yet, people do also find incongruity interesting and are motivated to engage cognitively with the product and its incongruity when the level of incongruity does not exceed their cognitive abilities (Meyers-levy and Tybout 1989). Thus, many findings indicate that people like a product more when it is slightly incongruent to an activated schema rather than products that are highly congruent or highly incongruent to an activated schema (Mandler 1982; Meyers-levy and Tybout 1989). However, it has also been shown that this nonmonotonic effect disappears when consumers are knowledgeable, as consumers are about a common human schema (Aggarwal and McGill 2007). Hence, we propose that congruent anthropomorphic product stimuli increase consumers' liking of the product and consumers are more likely to evaluate the product positively (Aggarwal and McGill 2007; Peracchio and Tybout 1996). We hypothesize:

H2: Given that anthropomorphism is indeed perceived as being congruent with autonomous products (H1), anthropomorphism should, in turn, increase the liking of autonomous products.

However, since the "schema concept refers to cognitive structures of organized prior knowledge, abstracted from experience with specific instances" (Fiske and Linville 1980, p. 543), the schema of consumers, who have prior experience with autonomous products, might differ to the schema of consumers, who do not have such experience. There is indeed some evidence that the evaluation of products 
differs between experts and novices (Hekkert et al. 2003). In general, consumer satisfaction is a function of expectation and expectancy disconfirmation (Oliver 1980). This corresponds to prior findings that consumers, who have had prior experience with technology, rated a robot with an anthropomorphic design less favorably than consumers, who have not had such experience (Goudey and Bonnin 2016). In detail, when mothers were asked about the intention to use a low-, medium-, or high-anthropomorphized robot as a companion for their children, intentions were moderated by the mothers' prior technology experience (Internet use through smartphones was used as a proxy for experience with technolgy): Mothers with more experience reported a higher usage intention for the robot with medium anthropomorphic design in comparison to the robot with high anthropomorphic design. When mothers had no prior technology experience, in contrast, they preferred the high-antrophomorphized over the medium-anthropomorphized robot (Goudey and Bonnin 2016). Based on these seminal findings, we hypothesize the following:

H3: Anthropomorphism increases the liking of autonomous products only if consumers have no prior experience with the product.

Moreover, of particular interest for marketers is the question of whether changing the product's description or appearance increases consumers' purchase intentions. To evaluate purchase intentions, several researches have applied the theory of reasoned action (Bosnjak et al. 2006; Kang and Lakshmanan 2017; Summers et al. 2006). The theory of reasoned action (Fishbein and Ajzen 1975) explains the intention of showing a specific behavior (e.g., buying a product) as a function of an individual's attitude toward the behavior and subjective norms associated with the behavior. Intention, as a central aspect in the model, describes "how hard people are willing [...] to perform the behavior" (Ajzen 1991, p. 181). Following this perspective, purchase intention describes how "hard" people are willing to buy a product. Since our research proposes that anthropomorphized autonomous products are liked more than non-anthropomorphized autonomous products, we also hypothesize:

H4: Increased liking of anthropomorphized autonomous products increases the purchase intention of this product.

Finally, of course autonomous products perform certain tasks, e.g., a vacuum cleaner cleans the floor and a lawn mower mows the grass. When consumers evaluate an object's performance, this is subject to biases (Wherry and Bartlett 1982) and influenced by affects (Abelson et al. 1982; Bower 1987; Clark 1982; Isen et al. 1985). Indeed, liking has been emphasized as a major influencing factor for the evaluation of performances and outcomes (Cardy and Dobbins 1986; Dipboye 1985). Therefore, we additionally hypothesize:

H5: An increased liking of an anthropomorphized autonomous product will, in turn, also increase the evaluation of the outcome obtained by the autonomous product, e.g., how well the floor is cleaned and the lawn is mowed.

We conducted three studies to test our hypotheses. Studies $1 \mathrm{a}$ and $1 \mathrm{~b}$ test hypothesis 1 . Study 2 tests hypotheses $2-5$. 


\section{Study 1}

The goal of our first study was to determine whether anthropomorphism is perceived to be more congruent with autonomous versus manual products. This allows us to test the hypothesis that consumers associate autonomous products more than manual products with a human schema.

It is a common approach to manipulate anthropomorphism on the basis of given traits (e.g., ambitious, ignorant, unemotional), which are perceived as more or less human (Ferrari et al. 2016; Haslam et al. 2005; Loughnan and Haslam 2007). Note, however, that human traits might be perceived as more positive than nonhuman traits in general (Haslam 2006). When autonomous products are perceived as more positive than manual products as well (Rijsdijk and Hultink 2003), trait valence would confound the effect of human (vs. nonhuman) traits on congruity perceptions. Even when statistically controlling for trait valence when determining the effect of the traits' anthropomorphic character on congruity, multicollinearity of the predictors could be an issue when trait valence and anthropomorphic character are highly correlated (Farrar and Glauber 1967). Therefore, we aimed to use both human and nonhuman trait stimuli that are perceived as positive or negative, respectively. Traits were selected based on the results of a pilot study.

In Study 1b, we tested whether anthropomorphic product descriptions are more congruent with autonomous products than with non-anthropomorphized products. Thus, we aimed to conceptually replicate the findings of Study 1a and show how to apply these results in a consumer-relevant context. In Study 1b, we therefore manipulated anthropomorphism in a textual way by using product descriptions that differ in the speaker's perspective; they were either from the first-person perspective, as if the product was speaking (e.g., "I work in households."), or from the third-person perspective (e.g., "It works in households."). This approach represents a common manipulation of anthropomorphism (Aggarwal and McGill 2007; Touré-Tillery and McGill 2015). To test the generalizability of the hypothesis, we used two different product categories in both sub-studies, i.e., vacuum cleaner and car.

\subsection{Study 1a}

\subsubsection{Pilot Study}

Participants $\left(N=36\right.$ English speaking individuals; $M_{\text {age }}=30.64, S D=10.61,77.8 \%$ female) were recruited via the online recruitment platform Prolific Academic, which has been shown to produce high-quality response data (Peer et al. 2016). They evaluated 23 preselected traits with regard to both perceived anthropomorphic character (human vs. non-human) and valence (positive vs. negative). To enforce one decision for every trait, the evaluation was conducted on the basis of a four-category matrix representing the possible combinations of the characteristics of the two dimensions, i.e., category 1: positive and human; 2: negative and human; 3: negative and nonhuman; 4: positive and non-human. Participants had to select one of the four categories for each trait (see Table A.1 in the supplementary material). Afterwards, for 
each category, participants were asked to rank the selected traits in relation to which traits fit this category best.

For each of the 23 traits, we analyzed which category the participants have chosen most (see Table A.2 in the supplementary material). Thereby, we got a selection of traits representing every category. To reduce this selection to the two best fitting traits, we additionally calculated the ranking average and chose the two traits with the highest ranking when there were more than two traits predominantly selected in a given category.

We obtained "pleasant" (percentage of participants who selected this category: $80.6 \%)$ and "supportive" (97.2\%) for category 1 (positive and human), "aggressive" and "stubborn" (both 88.9\%) for category 2 (negative and human), "intractable" $(50.0 \%)$ and "lifeless" $(52.8 \%)$ for category 3 (negative and non-human), and "multifunctional" (63.9\%) and "convenient" $(55.6 \%)$ for category 4 (positive and nonhuman).

\subsubsection{Methods}

Participants, Design, and Procedure For the main study, we gathered responses of $N=85$ English speaking participants $\left(M_{\text {age }}=33.02, S D=9.53,74.1 \%\right.$ female $)$ via Prolific Academic. To assure high quality of the data, we assessed participants' completion time and their attention by including attention-check items (Oppenheimer et al. 2009). Participants provided informed consent that they would only be eligible for a flat-fee payment if their completion time was within reasonable and predefined boundaries as well as if they passed the attention checks; they also agreed to their responses being used for scientific purposes.

We applied a within-subjects design, that is, participants rated the eight traits as selected in the pilot study (pleasant, supportive, aggressive, stubborn, intractable, lifeless, multifunctional, and convenient) in a randomized order. At the beginning of the study, participants received a short introduction about and definition of autonomous products (including exemplary pictures) to make sure that all participants had common knowledge about autonomous products and the main differences to manual products (see Table A.3 in the supplementary material). This description covered two kinds of autonomous products (lawn mower, vacuum cleaner) to ensure that participants think about autonomous products in a general way and not only about one specific product category.

Measures For each trait, participants answered questions about the anthropomorphic character, valence, and congruity with autonomous versus manual products. In detail, we measured anthropomorphic character with a semantic differential asking for: "Please rate, whether the word '[...]' fits better to the characteristics of 'objects' or 'human beings'. The word fits better to ...". Anchor points of the seven-point semantic differential were "very much better to objects", "much better to objects", "slightly better to objects", and "neutral" as well as "slightly better to human beings", "much better to human beings", and "very much better to human beings". We also measured valence on a seven-point semantic differential ranging from "extremely negative" to "extremely positive", where "neutral" represented the middle of the 
differential. Finally, congruity with autonomous versus manual products was measured on a seven-point semantic differential, too. We asked: "Please rate, whether the word '[...]' fits better to the characteristics of a 'manual' or an 'autonomous' product. The word fits better to ....". The anchor points of the seven-point semantic differential have been "very much better to manual products", "much better to manual products", "slightly better to manual products", and "neutral" as well as "slightly better to autonomous products", "much better to autonomous products", and "very much better to autonomous products".

\subsubsection{Results}

Because each participant rated all traits, trait ratings are nested within participants. To account for the interrelated error terms of responses from the same participant, we predicted perceptions of congruity using a mixed-effects regression. Perceived anthropomorphic character and valence of each trait were entered as fixed effects and the participant was modeled as a random effect (random intercept model; Pinheiro and Bates 2000).

On average, the traits were perceived to be somewhat more congruent with autonomous than with manual products, $M=4.36, S E=1.59$; scale: [1, 7]. More importantly and supporting $\mathrm{H} 1$, we found that with increasing values of a humanlike description of a trait, i.e., high anthropomorphic character, perceived congruity with autonomous products increases as well, $B=0.09, S E=0.03, p<0.001$. Traits which are perceived as more positive were also seen as more congruent with autonomous products, $B=0.19, S E=0.03, p<0.001$. Note that, as intended by the selection of traits via the pilot study, the within-subjects correlation of anthropomorphic character and valence was positive but weak $(r=0.11)$. Hence, multicollinearity of the predictors was no issue of concern. Moreover, the results remain virtually the same when controlling for participants' age and gender.

\subsection{Study $1 b$}

\subsubsection{Methods}

Participants, Design, and Procedure Study 1b was conducted online and gathered responses of $N=121$ English speaking participants $\left(M_{\text {age }}=33.12, S D=11.85\right.$, $57.9 \%$ female) via Prolific Academic. As in Study 1a, we assessed participants' completion time and their attention by including attention-check items. Payment conditions have also been the same and participants agreed to their responses being used for scientific purposes.

We applied a 2 (product description: first-person perspective vs. third-person perspective) $\times 2$ (product category: vacuum cleaner vs. car) between-subjects design. Every participant was randomly assigned to one of the described conditions, in which (s)he received four product descriptions in a randomized order, e.g., first-person perspective for car: "I am a car", "I drive on the streets", "I transport passengers to their destination", "I work with gasoline", and "I use my power to accelerate". 
Measures We measured perceived anthropomorphic character and perceived congruity to autonomous or manual products with the same measures as in Study 1a.

\subsubsection{Results}

In a first step, we tested whether the manipulation of anthropomorphism was successful. As intended, product descriptions (Cronbach's $\alpha=0.72$ ) using the first-person perspective were perceived as more anthropomorphic than product descriptions using the third-person perspective both for the vacuum cleaner, $M_{\text {first-person }}=3.55$, $S D=1.34$ vs. $M_{\text {third-person }}=2.48, S D=0.85 ; F(1,61)=13.70, p<0.001, f=0.48$, and the car, $M_{\text {first-person }}=3.30, S D=1.25$ vs. $M_{\text {third-person }}=2.63, S D=1.19 ; F(1,58)=4.45$, $p=0.039, f=0.29$.

To test our hypothesis, we performed an analysis of variance (ANOVA), using product description (first-person perspective vs. third-person perspective) and product category (vacuum cleaner vs. car) as predictors of perceived congruity to autonomous vs. manual products. Supporting H1, participants perceived anthropomorphic (first-person) product descriptions more congruent with autonomous products than non-anthropomorphic (third-person) product descriptions, $M_{\text {first-person }}=4.42$, $S D=1.06$ vs. $M_{\text {third-person }}=4.04, S D=0.95 ; F(1,120)=4.67, p=0.033, f=0.19$. We find no effect of product category on perceived congruity, $M_{\text {vacuum cleaner }}=4.11$, $S D=1.05$ vs. $M_{\text {car }}=4.37, S D=0.98 ; F(1,120)=2.26, p=0.136, f=0.13$. Moreover, the interaction effect of product category and product description is also not significant, $F(1,120)<1$.

\subsection{Discussion}

We provide seminal support for hypothesis 1 . Study 1a demonstrates that more humanlike traits are perceived as more congruent with the description of autonomous (vs. manual) products, irrespective of the valence of the trait. Study $1 \mathrm{~b}$ provides additional support for hypothesis 1 , by demonstrating that participants perceive anthropomorphic product descriptions to be more congruent with autonomous products than with manual products. This effect is independent of the product category. However, it is not clear whether anthropomorphic descriptions and displays of autonomous products have an impact on product liking, purchase intention, and product (performance) evaluations. This was addressed in Study 2.

\section{Study 2}

In Study 2, we build on the previous findings that anthropomorphism is perceived as more congruent with autonomous products than with manual products. As displayed in Fig. 1, we investigate whether increasing anthropomorphism of autonomous products also increases product liking (direct effect) and, in turn, purchase intention and evaluation of the product's outcomes (indirect effects via product liking).

In the context of autonomous products, the evaluation of an obtained outcome is of particular interest. After all, autonomous products are independent in their 


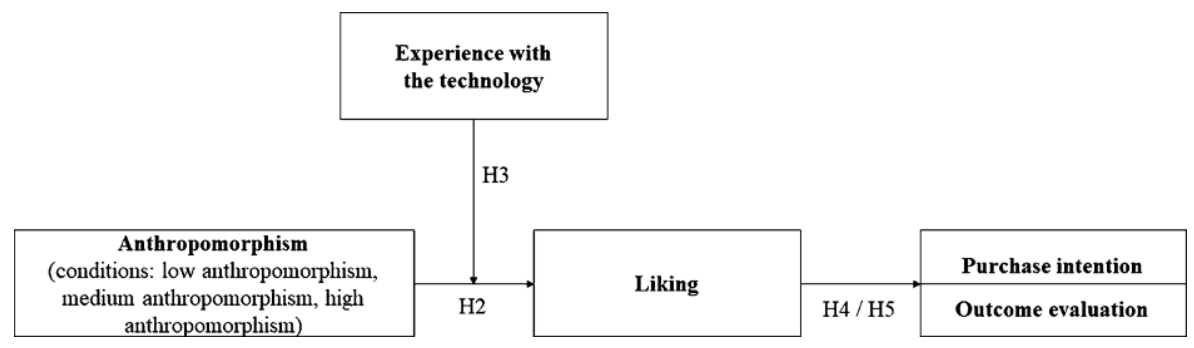

Fig. 1 Research model tested in Study 2

movement and/or decision-making and fulfill their tasks self-reliantly. Hence, an outcome produced by an autonomous product should be attributed to the autonomous product, since no interference with human operators takes place. Whether these outcomes are evaluated more positively, when the product is perceived as more anthropomorphic has important consequences for the consumers' post-purchase and long-term satisfaction with the product.

Study 2 was conducted as a lab experiment. We manipulated anthropomorphism of a real autonomous vacuum cleaner at different levels. Study 1 manipulated anthropomorphism in a textual way. We use this approach again, but extend it by adding a visual manipulation in one of three conditions. Thus, we complement our first two studies as well as previous research (Aggarwal and McGill 2007; Kim and McGill 2011; Landwehr et al. 2011) by investigating the effects of anthropomorphism of an actual physical product.

\subsection{Methods}

Participants, Design, and Procedure Participants were $N=172$ students $\left(M_{\text {age }}=24.40, S D=3.61,33.7 \%\right.$ female $)$ of a large German university. Participants were registered at the university's participant pool and were invited via the online registration software ORSEE (Greiner 2015). They received a flat-fee compensation of five Euro for participation in the 20-minute experiment. There were fifty experimental sessions with one to four participants each, which took place on six separate days.

We applied a one-factorial between-subjects design. Experimental sessions were randomly assigned to one of three experimental conditions: In the low anthropomorphism condition, participants saw a standard autonomous vacuum cleaner, which was labeled with a fictitious technical name, i.e., "KX903". In the medium anthropomorphism condition, the vacuum cleaner had the same optical appearance, but was labeled with an anthropomorphic name, i.e., "Bob". Finally, in the high anthropomorphism condition, the vacuum cleaner had the anthropomorphic name "Bob", too, and additionally was dressed in a custom-built anthropomorphic cover (visual manipulation, see Fig. 2). Manipulating anthropomorphism by name and design has been proven successful in prior research (Aggarwal and McGill 2007; Kim and McGill 2011; e.g., Vaes et al. 2016; Waytz et al. 2014). 


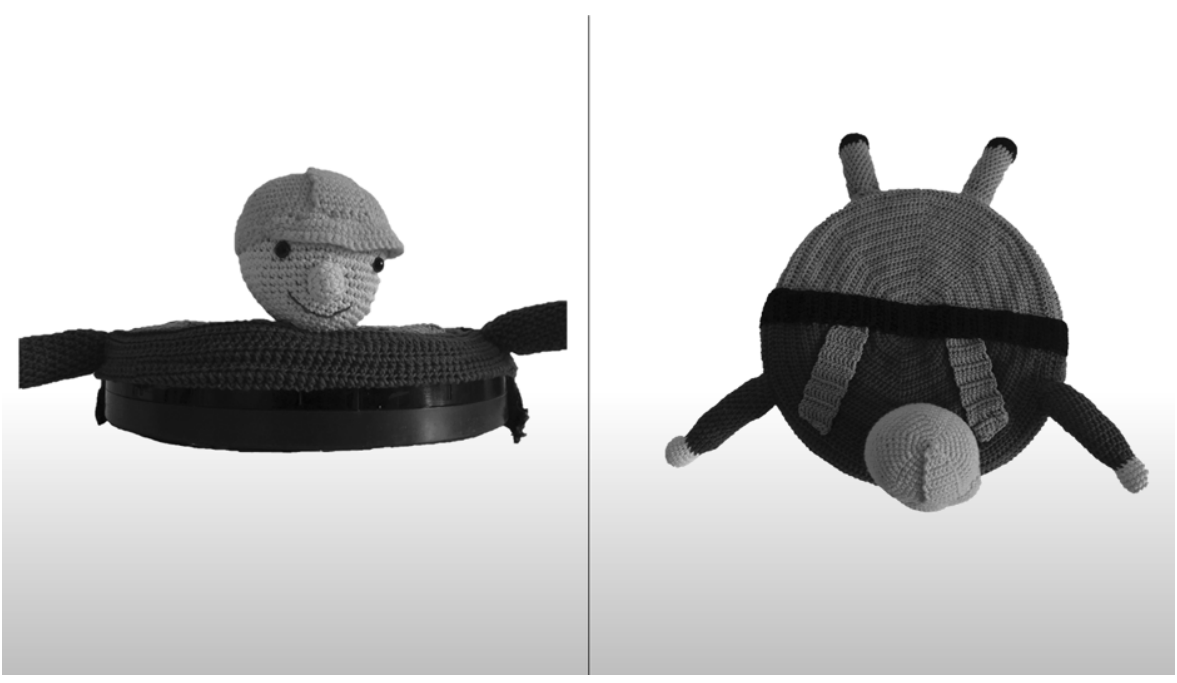

Fig. 2 Vacuum cleaner dressed in a custom-built anthropomorphic cover as used in the high anthropomorphism condition in Study 2
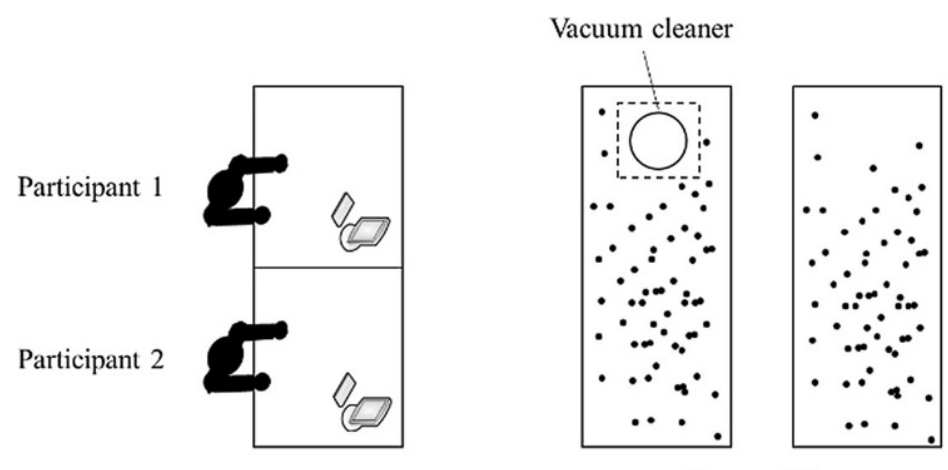

Study director

Strewn tables

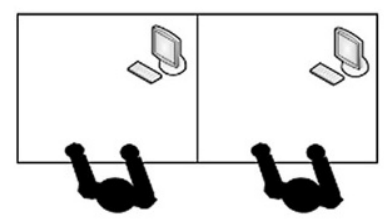

Participant 3 Participant 4

Fig. 3 Ground plot of the lab composition in Study 2. Note. Vacuum cleaner was covered at the beginning of each experimental session 
The procedure was as follows: Having arrived at the laboratory, participants were randomly seated at one of four computer stations (for the experimental set-up, see Fig. 3). In the middle of the room, we placed two equally sized tables, which had been identically strewn with finely ground bark mulch. On one of the tables, we placed the vacuum cleaner, which was covered by a blanket at the beginning of every experimental session. Thus, at the beginning of the study, participants could not see the vacuum cleaner and were consequently not influenced by its appearance.

The study consisted of three parts: In the first part (vacuum cleaner covered), participants answered questions about their current mood. After all participants had completed this part, the study director uncovered the vacuum cleaner and participants had to proceed with the second part of the study. Here, they received a description of the product from a first-person perspective (for a stimuli overview by condition, see Table B.1 in the supplementary material):

"Hi my name's [KX903/Bob] and I'm a household robot. I'm here on the table right beside you and you can take your time having a look at me. I'd like to ask you to make some judgments about my appearance. Afterwards, I'll start to clean the surface of the table for $1 \mathrm{~min}$ and then I'll ask you to evaluate me and my performance. Now, please take an unhurried look at me and tell me what you think."

After reading this description and having the possibility of inspecting the vacuum cleaner, participants answered questions about perceived anthropomorphism as well as product liking. In the third part, the experimenter started the vacuum cleaner, which cleaned the table for exactly one minute (the second table was not cleaned but served as a comparison to the other table). Afterwards, the vacuum cleaner was switched off, the experimenter removed it from the table and asked the participants to proceed with the survey. Participants answered questions about their mood again, evaluated the outcome, i.e., how well the table had been cleaned, and stated their purchase intention regarding the vacuum cleaner. When all participants had completed these questions, they were paid and allowed to leave.

Measures In the first and third parts, we measured participants' mood with four items on a five-point semantic differential proposed by Allen and Janiszewski (1989), requesting: "Please rate your current mood". The anchor points of the items were "Good/Bad", "Pleasant/Unpleasant", "Happy/Sad", and "Positive/Negative".

As a manipulation check of the independent variable, we assessed perceived product anthropomorphism in the second part using the three-item scale of Kim and McGill (2011), e.g., "[KX903/Bob] seems to have free will." on seven-point scales from $1=$ "strongly disagree" to $7=$ "strongly agree" $(\alpha=0.85)$. Moreover, product liking was measured with the ten-item scale of Carroll and Ahuvia (2006), e.g., "[KX903/Bob] is a wonderful product", on seven-point scales from $1=$ "strongly disagree" to $7=$ "strongly agree" $(\alpha=0.91)$.

In the third part, purchase intention was measured with three items proposed by Lepkowska-White et al. (2003), e.g., "If I were looking for this type of product, the likelihood of my purchasing [KX903/Bob] would be high." on seven-point scales from $1=$ "strongly disagree" to $7=$ "strongly agree" $(\alpha=0.94)$. Outcomes were evaluated using four items from Tsiros and Mittal (2000), e.g., "I am happy with [KX903's/Bob's] performance." on seven-point scales from $1=$ "strongly disagree" 
to $7=$ "strongly agree" $(\alpha=0.87)$. Lastly, we asked for demographics as well as prior experience with the technology: "Do you already have experience with robotic vacuum cleaners?" Due to the overall high internal consistency of the items for all scales, we used the respective mean values in our analyses (see Table B.2 in the supplementary material for a complete list of items).

Table 2 Moderated mediation analysis for the effects of product anthropomorphism on the dependent variables (Study 2)

\begin{tabular}{|c|c|c|c|c|c|c|c|c|}
\hline \multirow[b]{2}{*}{ Test } & \multicolumn{4}{|c|}{$\begin{array}{l}\text { No prior experience } \\
(n=136)\end{array}$} & \multicolumn{4}{|c|}{$\begin{array}{l}\text { Prior experience } \\
(n=36)\end{array}$} \\
\hline & $B$ & $S E$ & \multicolumn{2}{|c|}{$95 \%$ BC CI } & $B$ & $S E$ & \multicolumn{2}{|c|}{$95 \% B C C I$} \\
\hline \multicolumn{9}{|c|}{ Effects of independent variable on mediator } \\
\hline $\begin{array}{l}\text { Medium anthropomor- } \\
\text { phism } \rightarrow \text { Liking }\end{array}$ & 0.01 & 0.22 & -0.43 & 0.45 & -0.04 & 0.41 & -0.85 & 0.77 \\
\hline $\begin{array}{l}\text { High anthropomor- } \\
\text { phism } \rightarrow \text { Liking }\end{array}$ & 0.56 & 0.21 & 0.15 & 0.98 & -0.30 & 0.39 & -1.07 & 0.48 \\
\hline \multicolumn{9}{|c|}{ Effects of independent variable on dependent variables via mediator } \\
\hline $\begin{array}{l}\text { Medium anthropomor- } \\
\text { phism } \rightarrow \text { Liking } \rightarrow \text { Purcha } \\
\text { intention }\end{array}$ & 0.05 & 0.10 & -0.20 & 0.19 & -0.02 & 0.21 & -0.45 & 0.38 \\
\hline $\begin{array}{l}\text { High anthropomor- } \\
\text { phism } \rightarrow \text { Liking } \rightarrow \text { Purcha } \\
\text { intention }\end{array}$ & ise $^{0.28}$ & 0.13 & 0.06 & 0.58 & -0.15 & 0.20 & -0.56 & 0.25 \\
\hline $\begin{array}{l}\text { Medium anthropomor- } \\
\text { phism } \rightarrow \text { Liking } \rightarrow \text { Outcor } \\
\text { evaluation }\end{array}$ & ne & 0.06 & -0.13 & 0.13 & -0.01 & 0.13 & -0.31 & 0.22 \\
\hline $\begin{array}{l}\text { High anthropomor- } \\
\text { phism } \rightarrow \text { Liking } \rightarrow \text { Outcor } \\
\text { evaluation }\end{array}$ & ne & 0.14 & 0.04 & 0.43 & -0.10 & 0.14 & -0.42 & 0.15 \\
\hline \multicolumn{9}{|c|}{ Effects of independent variable on dependent variables } \\
\hline $\begin{array}{l}\text { Medium anthropo- } \\
\text { morphism } \rightarrow \text { Purchase } \\
\text { intention }\end{array}$ & -0.12 & 0.28 & -0.68 & 0.44 & 0.24 & 0.53 & -0.80 & 1.28 \\
\hline $\begin{array}{l}\text { High anthropomor- } \\
\text { phism } \rightarrow \text { Purchase } \\
\text { intention }\end{array}$ & 0.32 & 0.28 & -0.22 & 0.87 & -0.56 & 0.51 & -1.57 & 0.45 \\
\hline $\begin{array}{l}\text { Medium anthropomor- } \\
\text { phism } \rightarrow \text { Outcome } \\
\text { evaluation }\end{array}$ & -0.15 & 0.26 & -0.66 & 0.36 & 0.19 & 0.47 & -0.75 & 1.13 \\
\hline $\begin{array}{l}\text { High anthropomor- } \\
\text { phism } \rightarrow \text { Outcome } \\
\text { evaluation }\end{array}$ & -0.12 & 0.25 & -0.61 & 0.38 & -0.29 & 0.47 & -1.21 & 0.63 \\
\hline
\end{tabular}

Note. Mediation analyses were conducted separately for each of the dependent variables, i.e., purchase intention and outcome evaluation. 95\% BC CI: bias-corrected 95\% confidence intervals based on 10,000 bootstrap-iterations. Samples sizes of three different conditions: Low anthropomorphism $(n=51)$, reference group; medium anthropomorphism $(n=57)$; high anthropomorphism $(n=64)$ 
Table 3 Means and standard deviations of product liking, purchase intention, and outcome evaluation (Study 2)

\begin{tabular}{|c|c|c|c|c|c|c|c|c|}
\hline & \multicolumn{4}{|c|}{ No prior experience } & \multicolumn{4}{|c|}{ Prior experience } \\
\hline & & $\begin{array}{l}\text { Product } \\
\text { liking }\end{array}$ & $\begin{array}{l}\text { Purchase } \\
\text { intention }\end{array}$ & $\begin{array}{l}\text { Outcome } \\
\text { evalua- } \\
\text { tion }\end{array}$ & & $\begin{array}{l}\text { Product } \\
\text { liking }\end{array}$ & $\begin{array}{l}\text { Purchase } \\
\text { intention }\end{array}$ & $\begin{array}{l}\text { Outcome } \\
\text { evalua- } \\
\text { tion }\end{array}$ \\
\hline & $n$ & $M(S D)$ & $M(S D)$ & $M(S D)$ & $n$ & $M(S D)$ & $M(S D)$ & $M(S D)$ \\
\hline $\begin{array}{l}\text { Low an- } \\
\text { thropomor- } \\
\text { phism }\end{array}$ & 46 & $\begin{array}{l}2.46 \\
(1.06)\end{array}$ & $\begin{array}{l}2.91 \\
(1.32)\end{array}$ & $\begin{array}{l}3.35 \\
(1.29)\end{array}$ & 5 & $\begin{array}{l}3.48 \\
(1.36)\end{array}$ & $\begin{array}{l}4.07 \\
(1.30)\end{array}$ & $\begin{array}{l}3.85 \\
(1.62)\end{array}$ \\
\hline $\begin{array}{l}\text { Medium } \\
\text { anthropo- } \\
\text { morphism }\end{array}$ & 43 & $\begin{array}{l}2.83 \\
(0.97)\end{array}$ & $\begin{array}{l}2.95 \\
(1.50)\end{array}$ & $\begin{array}{l}3.04 \\
(1.50)\end{array}$ & 14 & $\begin{array}{l}2.85 \\
(1.18)\end{array}$ & $\begin{array}{l}3.62 \\
(1.61)\end{array}$ & $\begin{array}{l}3.77 \\
(1.39)\end{array}$ \\
\hline $\begin{array}{l}\text { High an- } \\
\text { thropomor- } \\
\text { phism }\end{array}$ & 47 & $\begin{array}{l}3.20 \\
(1.39)\end{array}$ & $\begin{array}{l}3.26 \\
(1.63)\end{array}$ & $\begin{array}{l}3.09 \\
(1.39)\end{array}$ & 17 & $\begin{array}{l}2.72 \\
(1.20)\end{array}$ & $\begin{array}{l}3.18 \\
(1.85)\end{array}$ & $\begin{array}{l}3.50 \\
(1.40)\end{array}$ \\
\hline
\end{tabular}

\subsection{Results}

In a first step, we tested differences in participants' perceived product anthropomorphism in the three experimental conditions. As expected, participants perceived the lowest anthropomorphism when the product was described with a technical name, followed by the condition where the product was described with a human name, and with the highest ratings when the product was described with a human name and a humanlike appearance, $M_{\text {low anthropomorphism }}=1.78, S D=1.07$ vs. $M_{\text {medium anthropomorphism }}=2.24, S D=1.50$ vs. $M_{\text {high anthropomorphism }}=2.73, S D=1.57 ; F(2$, $172)=6.42, p=0.002, f=0.28$. Hence, the manipulation proved to be successful.

To test the hypotheses, we conducted mediation and moderation analyses using maximum-likelihood estimations of indirect effects and reporting the corresponding 95\% bias-corrected confidence intervals (95\% BC CIs) based on 10,000 bootstrapiterations (see Table 2). The independent variable, i.e., anthropomorphism of the vacuum cleaner, was used as a multi-categorical predictor, as suggested by Hayes and Preacher (2014).

Table 3 provides the mean values of the dependent variables product liking, purchase intention, and outcome evaluation by experimental condition and prior experience with the technology. Supporting H2, participants liked the autonomous product more when it was anthropomorphized. Although this tendency exists for both the medium anthropomorphism and the high anthropomorphism conditions, we only find a significant difference regarding liking between the low anthropomorphism condition and the high anthropomorphism condition, $M_{\text {low anthropomorphism }}=2.56, S D=1.12$ vs. $M_{\text {medium anthropomorphism }}=2.83, S D=1.01$ vs. $M_{\text {high anthropomorphism }}=3.08, S D=1.35$; OLS regression low anthropomorphism vs. medium anthropomorphism: $B=0.27, S E=0.23$, $p=0.235 ; f=0.13$; OLS regression low anthropomorphism vs. high anthropomorphism: $B=0.51, S E=0.22, p=0.022 ; f=0.21$.

$\mathrm{H} 3$ proposes that the effect of anthropomorphism on liking is moderated by prior experience with the technology, i.e., anthropomorphism increases product liking only if the consumer has no prior experience with the technology. We analyzed this 


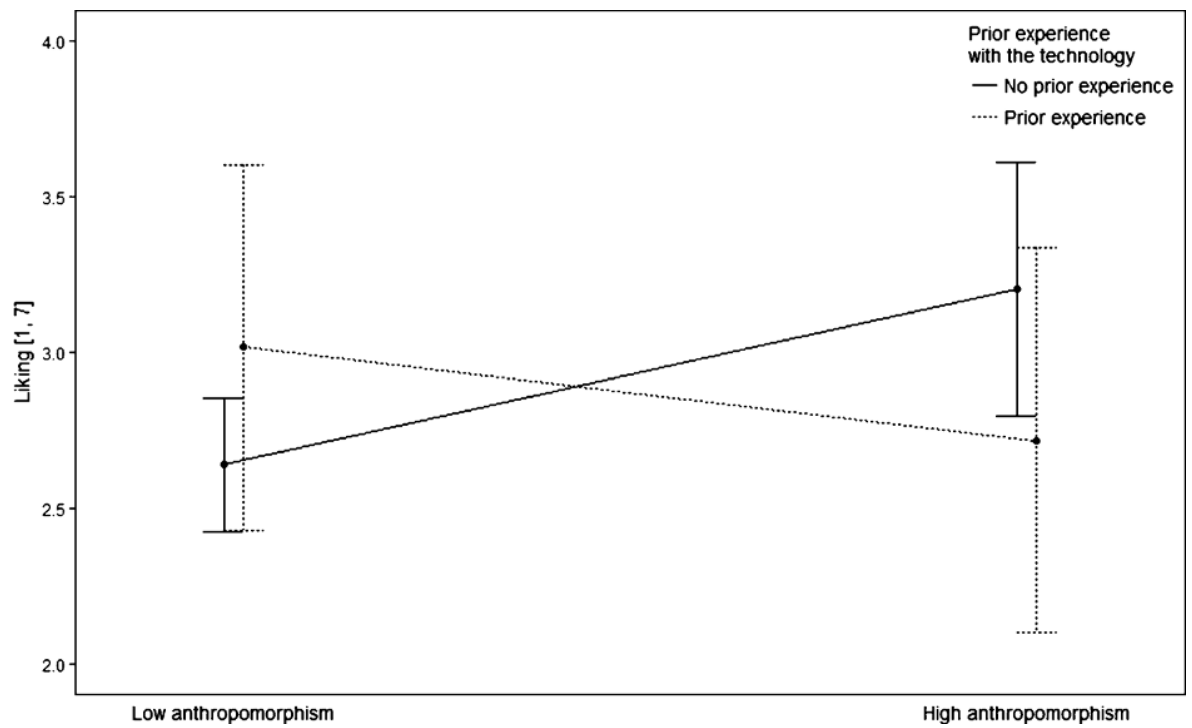

Fig. 4 Mean levels of product liking by product anthropomorphism and participants' prior experience with the technology in Study 2. Note. Error bars represent bootstrapped 95\% confidence intervals

relation by utilizing a moderation analysis (Preacher et al. 2007). As expected, in the high anthropomorphism condition we find an interaction effect of anthropomorphism and experience, $B=-0.86, S E=0.39, p=0.054$ (there was no significant interaction effect in the medium anthropomorphism condition, $B=-0.05, S E=0.46, p=0.914$ ). As displayed in Fig. 4, this effect indicates that for participants without prior experience with the technology $(n=136)$, high anthropomorphism increased product liking, $B=0.56, S E=0.21, p=0.008, f=0.243$ (the effect was not significant for the medium anthropomorphism condition, $B=0.01, S E=0.22, p=0.966 ; f=0.004)$. In contrast, for participants who had prior experience with the technology $(n=36)$, there was no positive effect of high anthropomorphism on product liking, in fact, there was even the tendency of a negative effect, $B=-0.30, S E=0.39, p=0.448$, $f=-0.123$.

To test hypotheses 4 and 5, we investigated whether product liking mediates the effect between product anthropomorphism and purchase intention as well as outcome evaluation, conditional on prior experience with the technology (moderated mediation as displayed in Fig. 1; Hayes 2015). As hypothesized, we find that product liking mediates the effect of product anthropomorphism on purchase intention, but only if participants had no prior experience with the technology. Again, the effect only reached significance for the high anthropomorphism condition, index of moderated mediation $=0.42 ; S E=0.25 ; 95 \%$ CI $[0.01,1.00]$ but not for the medium anthropomorphism condition, index of moderated mediation $=0.03 ; S E=0.23 ; 95 \%$ CI $[-0.43,0.48]$. In contrast, there were no significant indirect effects for participants who had prior experience with the technology, high anthropomorphism condition: $B=-0.15 ; S E=0.20 ; 95 \%$ CI $[-0.57,0.24]$, medium anthropomorphism condition: $B=-0.02 ; S E=0.20 ; 95 \%$ CI $[-0.44,0.38]$. 
Regarding the evaluation of the outcome obtained by the autonomous product, results are equivalent. That is, we find an indirect effect of perceived anthropomorphism via product liking on outcome evaluation, depending on prior experience with the technology. Again, while this tendency persists for both anthropomorphic product conditions when participants had no prior experience with the technology, it only reaches significance for the high anthropomorphism condition, index of moderated mediation $=-0.29 ; S E=0.18 ; 95 \% \mathrm{CI}[-0.71,-0.01]$, but not for the medium anthropomorphism condition, index of moderated mediation $=-0.02 ; S E=0.15 ; 95 \% \mathrm{CI}$ $[-0.34,0.25] .{ }^{1}$ For participants who had prior experience with the technology, none of the indirect effects was significant, high anthropomorphism condition: $B=-0.01$; $S E=0.14 ; 95 \%$ CI $[-0.41,0.15]$, medium anthropomorphism condition: $B=-0.01$; $S E=0.13 ; 95 \%$ CI $[-0.31,0.23]$.

Although these analyses suggest that the increased purchase intention and more positive outcome evaluation are due to an enhanced liking of autonomous products, alternatively, participants might just be in a more positive mood after seeing the anthropomorphized vacuum cleaner, which could then spillover onto purchase intentions and outcome evaluations. To exclude such an effect of emotional contagion (Hatfield et al. 1993) as a potentially confounding process, we also measured participants' mood before and immediately after participants have been confronted with the anthropomorphic product. A comparison of the mean values reveals no significant difference; hence, emotional contagion can be excluded as an alternative explanation for the observed effects, $M_{\text {mood before }}=2.00, S D=0.64$ vs. $M_{\text {mood after }}=2.01$, $S D=0.69, F(1,171)<1$.

\subsection{Discussion}

Results of Study 2 provide support for our hypotheses H2-H5. That is, we show that anthropomorphism implemented via a realistic (textual and visual) manipulation increases liking of an autonomous vacuum cleaner, given that participants had no prior experience with the technology. This, in turn, increases purchase intentions and the evaluation of outcomes obtained by the autonomous product.

Thus, anthropomorphizing autonomous products may have a positive effect in both the pre- and post-purchase phase, but only if consumers are not familiar with the technology. Note, however, that these effects only appeared in the high anthropomorphism condition, i.e., when the vacuum cleaner was labeled with a humanlike name and dressed in a humanlike cover.

\section{General Discussion}

There is an increasing prevalence of autonomous products in various domains. From a consumer perspective, autonomous products support the completion of a variety

\footnotetext{
${ }^{1}$ Note that although we find support for the hypothesized indirect effects, we do not find a total effect of experimental conditions on purchase intention or outcome evaluation (see Table 1). We further address this finding in the General Discussion.
} 
of tasks in everyday life. From a company perspective, they represent a promising future market (Executive Summary World Robotics 2019 Service Robots 2019). However, little is known about the psychological mechanisms underlying consumers' perceptions of autonomous products and its implications for marketers (Kim et al. 2016), i.e., how to describe and design autonomous products in order to increase consumers' pre- and post-purchase satisfaction (Kiesler and Goetz 2002).

\subsection{Theoretical Contribution}

Our research contributes to this important field of research of autonomous products (Rijsdijk and Hultink 2003; Schweitzer and van den Hende 2016; Waytz et al. 2014). In two online studies and one lab study, we identify anthropomorphism as a promising brick for market placement and the design of autonomous products, but we also show its limitations. Previous research revealed mixed effects regarding the impact of anthropomorphism on the perception of technology. We argue that autonomous products are perceived to be congruent with a human schema. In other words, anthropomorphized autonomous products correspond to consumers' expectations (Aggarwal and McGill 2007). In line with previous research on schema congruity as a basis for product evaluation (Aggarwal and McGill 2007; Mandler 1982; Meyers-levy and Tybout 1989; Peracchio and Tybout 1996; van den Hende and Mugge 2014), anthropomorphized autonomous products should be liked more than non-anthropomorphized autonomous products.

Our results support this relation, but we find an important limitation. When consumers have prior experience with the technology, anthropomorphism does not increase product liking, but could even decrease it. This result corresponds to previous findings, which have shown adverse effects of product anthropomorphism on the evaluation of intelligent products, depending on consumers' technological attitude and usage (Belk 2016; Goudey and Bonnin 2016). We interpret this finding such that consumers with prior experience with (non-anthropomorphized) autonomous products might have a predefined expectation about these products, which is mostly technical. Hence, these consumers have a schema of autonomous products, which is less congruent to a human schema and different to the schema of consumers without prior product experience.

In addition to the direct effect of anthropomorphism on product liking, we also find indirect effects on purchase intention and, interestingly, on the evaluation of outcomes obtained by the autonomous product. This indicates that anthropomorphizing autonomous products may not only have positive effect on the pre-purchase phase but also on the post-purchase phase.

\subsection{Managerial Implications}

Taking these effects together, our research provides novel insights about anthropomorphizing products in general, and autonomous products in particular (Złotowski et al. 2015). We suggest that marketers should seriously consider anthropomorphizing autonomous products, particularly in an early adoption phase, when many consumers have no personal experiences with autonomous products yet. Anthropo- 
morphizing autonomous products can help users to familiarize with the unknown technology faster and overcome adoption barriers in the first place. Based on the results of our studies, we outline some implications for managers and marketers of autonomous products.

We recommend managers to segment their customers based on their prior experience with autonomous products. That means, currently, the majority of potential customers is rather unexperienced with autonomous products. Our results indicate that anthropomorphized autonomous products lead to higher liking of first-time or less experienced customers. To provide an idea how such a "customization" based on customers' experience could work in practice, we suggest that companies could provide customers with the option to select an individual design and name of their product, e.g., when they order it online.

By targeting the less experienced customers, companies can anthropomorphize autonomous products in different ways, for example, products such as lawn mowers or vacuum cleaners can be advertised with a real name ("Bob") instead of just a technical description ("KX903"). The personification can be used in the promotion of the product and also in the product description. In addition to the name, the design of autonomous products can be adapted, for example adding a cute face or other decoration on the product surface.

\subsection{Limitations and Outlook}

Although our studies provide important insights for both theory and practice, there certainly exist some limitations and potential extensions for future research. Our research indicates that only inexperienced consumer may increase the liking of autonomous products when these are anthropomorphized. Yet, additionally, our results could partly be driven by experienced consumers decreasing their liking of the anthropomorphized product due to a decreased competence evaluation. Future research should therefore investigate in more detail how prior experience changes the perceptions of anthropomorphized products on different levels (e.g., liking, competence, autonomy). Relatedly, it is interesting to investigate how the preexisting schema of experienced consumers can be modified to make these people accessible for anthropomorphization of autonomous products, too. Moreover, the identification of further moderators (e.g., consumers' attitudes toward the product and their technological orientation) and their impact on the link between anthropomorphism and product liking appears a promising area for future research.

Additionally, in Study 2 we manipulated anthropomorphism by giving the autonomous robot a human name (medium anthropomorphism condition) and a human costume in combination with a human name (high anthropomorphism condition). Even though this manipulation is salient, the perceived anthropomorphism remains on a quite low absolute level ( $M_{\text {high anthropomorphism }}=3.08$ on a 7-point scale). However, stronger manipulations without restricting the functions of the investigated robots seem to be limited. But even when this would be possible, the prominent model of uncanny valley proposes that the relation between perceived anthropomorphism and liking is non-monotonic and an anthropomorphic design that is too strong may 
backfire at some point (Mori et al. 2012). Future research as well as companies should consider this when designing anthropomorphic domestic robots.

Finally, in Study 2 we do not find a significant effect of anthropomorphism on liking in the medium anthropomorphism condition. While the medium anthropomorphism manipulation was probably not strong enough to significantly influence liking, we would also like to emphasize the need to consider the main difference between the medium and the high anthropomorphic manipulation. That is, we used a textual and visual cue in the high anthropomorphism condition and a textual manipulation in the medium anthropomorphism condition. We find it interesting to further study the independent and joint effects of anthropomorphism manipulations in different (textual vs. visual) domains.

Finally, future research may extend and generalize our findings to other autonomous products. For instance, do consumers also evaluate a trip in an anthropomorphized autonomous car more positive than a trip in a non-anthropomorphized autonomous car?

\subsection{Conclusion}

Overall and without a doubt, an increasing amount of tasks and duties will be transferred from humans to autonomous products in the near future. It is important for the further diffusion and success of autonomous products to better understand consumers' perceptions of such products, affecting the way in which these products are advertised. Our research provides important insights into how to increase consumers' positive pre- and post-purchase perceptions of autonomous products by anthropomorphizing them.

Funding Open Access funding provided by Projekt DEAL.

Open Access This article is licensed under a Creative Commons Attribution 4.0 International License, which permits use, sharing, adaptation, distribution and reproduction in any medium or format, as long as you give appropriate credit to the original author(s) and the source, provide a link to the Creative Commons licence, and indicate if changes were made. The images or other third party material in this article are included in the article's Creative Commons licence, unless indicated otherwise in a credit line to the material. If material is not included in the article's Creative Commons licence and your intended use is not permitted by statutory regulation or exceeds the permitted use, you will need to obtain permission directly from the copyright holder. To view a copy of this licence, visit http://creativecommons.org/licenses/by/4. $0 /$.

\section{References}

Aaker, J.L. 1997. Dimensions of brand personality. Journal of Marketing Research 34(3):347-356. https:// doi.org/10.2307/3151897.

Abelson, R.P., D.R. Kinder, M.D. Peters, and S.T. Fiske. 1982. Affective and semantic components in political person perception. Journal of Personality and Social Psychology 42(4):619-630. https://doi. org/10.1037/0022-3514.42.4.619.

Aggarwal, P., and A.L. McGill. 2007. Is that car smiling at me? Schema congruity as a basis for evaluating anthropomorphized products. Journal of Consumer Research 34(4):468-479. https://doi.org/10.1086/ 518544.

Aggarwal, P., and A.L. McGill. 2012. When brands seem human, do humans act like brands? Automatic behavioral priming effects of brand anthropomorphism. Journal of Consumer Research 39(2):307-323. https://doi.org/10.1086/662614. 
Ajzen, I. 1991. The theory of planned behavior. Organizational Behavior and Human Decision Processes 50(2):179-211. https://doi.org/10.1016/0749-5978.

Allen, C.T., and C.A. Janiszewski. 1989. Assessing the role of contingency awareness in attitudinal conditioning with implications for advertising research. Journal of Marketing Research 26(1):30. https:// doi.org/10.2307/3172667.

BCG. 2017. Autonomous vehicle adoption study. https://www.bcg.com/de-de/industries/automotive/ autonomous-vehicle-adoption-study.aspx. Accessed 12 Dec 2017.

Beer, J.M., A.D. Fisk, and W.A. Rogers. 2014. Toward a framework for levels of robot autonomy in human-robot interaction. Journal of Human-Robot Interaction 3(2):74. https://doi.org/10.5898/JHRI.3. 2.Beer.

Bekey, G.A. 1998. Introduction. In Autonomous agents, Vol. 5, ed. G.A. Berkey. New York: Springer. https://doi.org/10.1093/0195150430.001.0001.

Belk, R. 2016. Understanding the robot: comments on Goudey and Bonnin (2016). Recherche et Applications En Marketing 31(4):83-90. https://doi.org/10.1177/2051570716658467.

Bosnjak, M., D. Obermeier, and T.L. Tuten. 2006. Predicting and explaining the propensity to bid in online auctions: a comparison of two action-theoretical models. Journal of Consumer Behaviour 5(2):102-116. https://doi.org/10.1002/cb.38.

Bower, G.H. 1987. Commentary on mood and memory. Behaviour Research and Therapy 25(6):443-455. https://doi.org/10.1016/0005-7967(87)90052-0.

Breazeal, C. 2003. Toward sociable robots. Robotics and Autonomous Systems 42(3/4):167-175. https:// doi.org/10.1016/S0921-8890(02)00373-1.

Broadbent, E., V. Kumar, X. Li, J. Sollers, R.Q. Stafford, B.A. MacDonald, and D.M. Wegner. 2013. Robots with display screens: a robot with a more humanlike face display is perceived to have more mind and a better personality. Plos One https://doi.org/10.1371/journal.pone.0072589.

Burgoon, J.K., J.A. Bonito, B. Bengtsson, C. Cederberg, M. Lundeberg, and L. Allspach. 2000. Interactivity in human-computer interaction: a study of credibility, understanding, and influence. Computers in Human Behavior 16(6):553-574. https://doi.org/10.1016/S0747-5632(00)00029-7.

Cardy, R.L., and G.H. Dobbins. 1986. Affect and appraisal accuracy: liking as an integral dimension in evaluating performance. Journal of Applied Psychology 71(4):672-678. https://doi.org/10.1037/ 0021-9010.71.4.672.

Carroll, B.A., and A.C. Ahuvia. 2006. Some antecedents and outcomes of brand love. Marketing Letters 17(2):79-89. https://doi.org/10.1007/s11002-006-4219-2.

Chandler, J., and N. Schwarz. 2010. Use does not wear ragged the fabric of friendship: thinking of objects as alive makes people less willing to replace them. Journal of Consumer Psychology 20(2):138-145. https://doi.org/10.1016/j.jcps.2009.12.008.

Clark, M.S. 1982. A role for arousal in the rink between feeling states, judgments, and behavior. In Affect and cognition, ed. M.S. Clark, S.T. Fiske, 263-290. Hillsdale, NJ: Erlbaum.

Dipboye, R.L. 1985. Some neglected variables in research on discrimination in appraisals. Academy of Management 10(1):116-127. https://doi.org/10.5465/AMR.1985.4277365.

Epley, N., A. Waytz, and J.T. Cacioppo. 2007. On seeing human: a three-factor theory of anthropomorphism. Psychological Review 114(4):864-886. https://doi.org/10.1037/0033-295X.114.4.864.

Executive Summary World Robotics. 2019. Service Robots. https://ifr.org/downloads/press2018/Executive_ Summary_WR_Service_Robots_2019.pdf. Accessed: 29 Oct 2019.

Fan, A., L. Laurie Wu, and A.S. Mattila. 2016. Does anthropomorphism influence customers' switching intentions in the self-service technology failure context? Journal of Services Marketing 30(7):713-723. https://doi.org/10.1108/JSM-07-2015-0225.

Farrar, D.E., and R.R. Glauber. 1967. Multicollinearity in regression analysis: the problem revisited. The Review of Economics and Statistics 49(1):92. https://doi.org/10.2307/1937887.

Ferrari, F., M.P. Paladino, and J. Jetten. 2016. Blurring human - machine distinctions: anthropomorphic appearance in social robots as a threat to human distinctiveness. International Journal of Social Robotics 8(2):287-302. https://doi.org/10.1007/s12369-016-0338-y.

Fishbein, M., and I. Ajzen. 1975. Belief, attitude, intention, and behavior: an introduction to theory and research. Reading, MA: Addison-Wesley.

Fiske, S.T., and P.W. Linville. 1980. What does the schema concept buy us? Personality and Social Psychology Bulletin 6(4):543-557. https://doi.org/10.1177/014616728064006.

Fournier, S., and C. Alvarez. 2012. Brands as relationship partners: warmth, competence, and in-between. Journal of Consumer Psychology 22(2):177-185. https://doi.org/10.1016/j.jcps.2011.10.003. 
Gong, L. 2008. How social is social responses to computers? The function of the degree of anthropomorphism in computer representations. Computers in Human Behavior 24(4):1494-1509. https://doi.org/ 10.1016/j.chb.2007.05.007.

Goudey, A., and G. Bonnin. 2016. Must smart objetcs look human? Study of the impact of anthropomorphism on the acceptance of companion robots. Recherche et Applications En Marketing 31(2):2-20. https://doi.org/10.1177/2051570716643961.

Gray, K., and D.M. Wegner. 2012. Feeling robots and human zombies: mind perception and the uncanny valley. Cognition 125(1):125-130. https://doi.org/10.1016/j.cognition.2012.06.007.

Greiner, B. 2015. Subject pool recruitment procedures: organizing experiments with ORSEE. Journal of the Economic Science Association 1(1):114-125. https://doi.org/10.1007/s40881-015-0004-4.

Hancock, P.A., D.R. Billings, K.E. Schaefer, J.Y.C. Chen, E.J. de Visser, and R. Parasuraman. 2011. A meta-analysis of factors affecting trust in human-robot interaction. Human Factors: The Journal of the Human Factors and Ergonomics Society 53(5):517-527. https://doi.org/10.1177/0018720811417254.

Haslam, N. 2006. Dehumanization: an integrative review. Personality and Social Psychology Review 10(3):252-264. https://doi.org/10.1207/s15327957pspr1003_4.

Haslam, N., P. Bain, L. Douge, M. Lee, and B. Bastian. 2005. More human than you: attributing humanness to self and others. Journal of Personality and Social Psychology 89(6):937-950. https://doi.org/10. 1037/0022-3514.89.6.937.

Hatfield, E., J.T. Cacioppo, and R.L. Rapson. 1993. Emotional contagion. Current Directions in Psychological Sciences 2(3):96-99. https://doi.org/10.1111/1467-8721.ep10770953.

Hayes, A.F. 2015. An index and test of linear moderated mediation. Multivariate Behavioral Research 50:1-22. https://doi.org/10.1080/00273171.2014.962683.

Hayes, A.F., and K.J. Preacher. 2014. Statistical mediation analysis with a multicategorical independent variable. British Journal of Mathematical and Statistical Psychology 67(3):451-470. https://doi.org/ 10.1111/bmsp.12028.

Hekkert, P., D. Snelders, and P.C.W. Wieringen. 2003. 'Most advanced, yet acceptable': typicality and novelty as joint predictors of aesthetic preference in industrial design. British Journal of Psychology 94(1):111-124. https://doi.org/10.1348/000712603762842147.

van den Hende, E.A., and R. Mugge. 2014. Investigating gender-schema congruity effects on consumers' evaluation of anthropomorphized products. Psychology \& Marketing 31(4):264-277. https://doi.org/ 10.1002/mar.20693.

Holzwarth, M., C. Janiszewski, and M.M. Neumann. 2006. The influence of avatars on Online consumer shopping behavior. Journal of Marketing 70:19-36.

Isen, A.M., M.M.S. Johnson, E. Mertz, and G.F. Robinson. 1985. The influence of positive affect on the unusualness of word associations. Journal of Personality and Social Psychology 48(6):1413-1426. https://doi.org/10.1037/0022-3514.48.6.1413.

Jörling, M., R. Böhm, and S. Paluch. 2019. Service robots: drivers of perceived responsibility for service outcomes. Journal of Service Research 22(4):404-420. https://doi.org/10.1177/1094670519842334.

Kang, E., and A. Lakshmanan. 2017. Role of executive attention in consumer learning with background music. Journal of Consumer Psychology 27(1):35-48. https://doi.org/10.1016/j.jcps.2016.03.003.

Kiesler, S., and J. Goetz. 2002. Mental models and cooperation with robotic assistants. CHI'O2 Extended Abstracts on Human Factors in Computing Systems https://doi.org/10.1145/506443.506491.

Kim, S., and A.L. McGill. 2011. Gaming with Mr. Slot or gaming the slot machine? Power, anthropomorphism, and risk perception. Journal of Consumer Research 38(1):94-107. https://doi.org/10.1086/ 658148.

Kim, S., R.P. Chen, and K. Zhang. 2016. Anthropomorphized helpers undermine autonomy and enjoyment in computer games. Journal of Consumer Research 43(2):282-302. https://doi.org/10.1093/jcr/ ucw016.

Koda, T., and P. Maes. 1996. Agents with faces: the effect of personification. In IEEE International Workshop on Robot and Human Communication, 189-194.

Landwehr, J.R., A.L. McGill, and A. Herrmann. 2011. It's got the look: the effect of friendly and aggressive "facial" expressions on product liking and sales. Journal of Marketing 75(3):132-146. https://doi.org/ 10.1509/jmkg.75.3.132.

Lee, S.L., I.Y.M. Lau, and Y.Y. Hong. 2011. Effects of appearance and functions on likability and perceived occupational suitability of robots. Journal of Cognitive Engineering and Decision Making 5(2):232-250. https://doi.org/10.1177/1555343411409829.

Lepkowska-White, E., T. Brashear, and M. Weinberger. 2003. A test of ad appeal effectiveness in Poland and the United States-The interplay of appeal, product, and culture. Journal of Advertising 32(3):57-67. 
Loughnan, S., and N. Haslam. 2007. Animals and androids. Psychological Science 18(2):116-122.

Mandler, G. 1982. The structure of value: accounting for taste. In Affect and Cognition: The 17th Annual Carnegie Symposium, ed. Margaret S. Clark, S.T. Fiske, 3-36. Hillsdale, NJ: Lawrence Erlbaum.

Mende, M., M.L. Scott, J. van Doorn, D. Grewal, and I. Shanks. 2019. Service robots rising: how humanoid robots influence service experiences and elicit compensatory consumer responses. Journal of Marketing Research https://doi.org/10.1177/0022243718822827.

Meyers-levy, J., and A.M. Tybout. 1989. Schema congruity as a basis for product evaluation. Journal of Consumer Research 16:39-55.

Mori, M., K.F. MacDorman, and N. Kageki. 2012. The uncanny valley. IEEE Robotics and Automation Magazine 19(2):98-100. https://doi.org/10.1109/MRA.2012.2192811.

Oliver, R.L. 1980. A cognitive model of the antecedents and consequences of satisfaction decisions. Journal of Marketing Research 17(4):460. https://doi.org/10.2307/3150499.

Oppenheimer, D.M., T. Meyvis, and N. Davidenko. 2009. Instructional manipulation checks: detecting Satisficing to increase statistical power. Journal of Experimental Social Psychology 45(4):867-872. https://doi.org/10.1016/j.jesp.2009.03.009.

Peer, E., S. Samat, L. Brandimarte, and A. Acquisti. 2016. Beyond the Turk: alternative platforms for crowdsourcing behavioral research. Journal of Experimental Social Psychology 70:153-163. https:// doi.org/10.1016/j.jesp.2017.01.006.

Peracchio, L.A., and A.M. Tybout. 1996. The moderating role of prior knowledge in schema-based product evaluation. Journal of Consumer Research 23(3):177-192. https://doi.org/10.1086/209476.

Pinheiro, J.C., and D.M. Bates. 2000. Extending the basic linear mixed-effects model. Methods. New York: Springer. https://doi.org/10.1007/b98882.

van Pinxteren, M.M.E., R.W.H. Wetzels, J. Rüger, M. Pluymaekers, and M. Wetzels. 2019. Trust in humanoid robots: implications for services marketing. Journal of Services Marketing 33(4):507-518. https://doi.org/10.1108/JSM-01-2018-0045.

Preacher, K.J., D.D. Rucker, and A.F. Hayes. 2007. Addressing moderated mediation hypotheses: theory, methods, and prescriptions. Multivariate Behavioral Research 42(1):185-227. https://doi.org/ $10.1080 / 00273170701341316$.

Rijsdijk, S., and E.J. Hultink. 2009. How today's consumers perceive tomorrow's smart products. Journal of Product Innovation Management https://doi.org/10.1111/j.1540-5885.2009.00332.x.

Rijsdijk, S.A., and E.J. Hultink. 2003. "Honey, have you seen our hamster?" Consumer evaluations of autonomous domestic products. Journal of Product Innovation Management 20(3):204-216. https:// doi.org/10.1111/1540-5885.2003003.

Schweitzer, F., and E. van den Hende. 2016. To be or not to be in thrall to the march of smart products. Psychology \& Marketing 33(10):830-842. https://doi.org/10.1002/mar.20920.

Summers, T.A., B.D. Belleau, and Y. Xu. 2006. Predicting purchase intention of a controversial luxury apparel product. Journal of Fashion Marketing and Management: An International Journal 10(4):405-419. https://doi.org/10.1108/13612020610701947.

Touré-Tillery, M., and A.L. McGill. 2015. Who or what to believe: trust and the differential persuasiveness of human and anthropomorphized messengers. Journal of Marketing 79(4):94-110. https://doi.org/ $10.1509 /$ jm.12.0166.

Tsiros, M., and V. Mittal. 2000. Regret: a model of its antecedents and consequences in consumer decision making. Journal of Consumer Research 26(4):401-417. https://doi.org/10.1086/209571.

Vaes, J., F. Meconi, P. Sessa, and M. Olechowski. 2016. Minimal humanity cues induce neural empathic reactions towards non-human entities. Neuropsychologia 89:132-140. https://doi.org/10.1016/ j.neuropsychologia.2016.06.004.

de Visser, E.J., S.S. Monfort, R. McKendrick, M.A.B. Smith, P.E. McKnight, F. Krueger, and R. Parasuraman. 2016. Almost human: anthropomorphism increases trust resilience in cognitive agents. Journal of Experimental Psychology: Applied 22(3):331-349. https://doi.org/10.1037/xap0000092.

Waytz, A., J. Cacioppo, and N. Epley. 2010a. Who sees human? Perspectives on Psychological Science 5(3):219-232. https://doi.org/10.1177/1745691610369336.

Waytz, A., J. Heafner, and N. Epley. 2014. The mind in the machine: anthropomorphism increases trust in an autonomous vehicle. Journal of Experimental Social Psychology 52:113-117. https://doi.org/10. 1016/j.jesp.2014.01.005.

Waytz, A., C.K. Morewedge, N. Epley, G. Monteleone, J.-H. Gao, and J.T. Cacioppo. 2010b. Making sense by making sentient: effectance motivation increases anthropomorphism. Journal of Personality and Social Psychology 99(3):410-435. https://doi.org/10.1037/a0020240.

Wherry, R.J., and C.J. Bartlett. 1982. The control of bias in ratings: a theory of rating. Personnel Psychology 35(3):521-551. https://doi.org/10.1111/j.1744-6570.1982.tb02208.x. 
Yogeeswaran, K., J. Złotowski, M. Livingstone, C. Bartneck, H. Sumioka, and H. Ishiguro. 2016. The interactive effects of robot anthropomorphism and robot ability on perceived threat and support for robotics research. Journal of Human-Robot Interaction 5(2):29. https://doi.org/10.5898/jhri.5.2. yogeeswaran.

Ziemke, T. 2008. On the role of emotion in biological and robotic autonomy. BioSystems 91(2):401-408. https://doi.org/10.1016/j.biosystems.2007.05.015.

Złotowski, J., D. Proudfoot, K. Yogeeswaran, and C. Bartneck. 2015. Anthropomorphism: opportunities and challenges in human-robot interaction. International Journal of Social Robotics 7(3):347-360. https://doi.org/10.1007/s12369-014-0267-6.

Publisher's Note Springer Nature remains neutral with regard to jurisdictional claims in published maps and institutional affiliations. 